\title{
ORIENTACIÓN PROFESIONAL Y SITUACIÓN SOCIO-ECONÓMICA EN ARGENTINA
}

\author{
CAREER GUIDANCE AND SOCIO-ECONOMIC SITUATION IN ARGENTINA
}

\author{
Andrea Ferrero* \\ Universidad Nacional de San Luis, Argentina
}

\begin{abstract}
RESUMEN
Este trabajo desarrolla las circunstancias bajo las cuales, desde sus inicios, la orientación profesional en Argentina se caracterizó por el hecho de contemplar especialmente las condiciones sociales de la población a la que dirigía su accionar. Efectivamente, la orientación profesional en este país estuvo básicamente relacionada con la detección de determinadas características personales y su relación con capacidades laborales específicas, dentro de un contexto en el cual los parámetros vinculados a aspectos sociales eran incluidos como uno de los articuladores fundamentales de dicha tarea. En Argentina es posible apreciar cómo, durante las últimas décadas, la exclusión social producto de los crecientes niveles de pobreza, la falta de oportunidades, el desempleo y los bajos niveles de educación, han introducido nuevas perspectivas en el panorama referido al proceso de orientación. Si bien éste históricamente ha contemplado los aspectos sociales y económicos, el elevado grado de dificultad estructural de la situación actual representa nuevos y complejos desafíos en la tarea de orientación. En este artículo se analiza el valor del orientador comprometido con factores socio-económicos de las poblaciones a las que dirige su tarea y la importancia que la responsabilidad social adquiere en estas circunstancias. Responsabilidad que incluso se encuentra avalada en Argentina, tanto por códigos de ética locales como por las propias competencias internacionales de la orientación profesional.
\end{abstract}

Palabras clave: orientación profesional - factores socio-económicos - ética - competencias profesionales - Argentina.

\section{ABSTRACT}

This work points out the circumstances in which, from its very beginning, professional guidance in Argentina has been specially characterized by taking into account the particular social situation of the individuals and populations to whom the specific practice was directed. In fact, professional guidance

\footnotetext{
* Andrea Ferrero. Doctora en Psicología. Docente e Investigadora de la Universidad Nacional de San Luis, Argentina. Directora del Proyecto: "Aspectos deontológicos y éticos de la formación de pregrado y de la práctica profesional de la psicología en Argentina. Estudio comparativo entre los países del Mercosur”, subsidiado por la Secretaría de Ciencia y Técnica de la Universidad Nacional de San Luis. Coordinadora de la Task Force "Etica y Deontología Profesional” de la Sociedad Interamericana de Psicología. E-mail: aferrero@unsl.edu.ar
} 
in this country was basically related to the detection of certain individual factors and its relation to specific labour abilities, within a context in which social matters' related factors were considered as a main axe to the future developing of the whole guidance process. In Argentina, during the last decades, social exclusion due to increasingly poverty index, the lack of opportunities, the unemployment and a low educational level, have introduced hard new conditions into careers advice. Even though this process has always considered social and economical matters, the high level structural complexity of current situation means new and hard challenges in the guidance task. This paper analyzes the central role of careers advisers' commitment to socio-economical circumstances of the individuals and population to whom the task is directed, and the importance of social responsibility involved through these circumstances. This responsibility is also supported in Argentina, not only by local ethics codes, but by professional international guidance competences as well.

Key words: careers advice - social and political issues - ethics - professional competences - Argentina.

\section{Introducción}

Desde mediados de siglo XX se distingue entre orientación profesional individual y colectiva. La primera de ellas apuntaba a que cada individuo se dedicara al trabajo más adecuado para sí, obteniendo el máximo rendimiento con el menor esfuerzo; la segunda propiciaba la adecuada distribución de la población trabajadora acorde a intereses y necesidades del país según un determinado plan económico y social (Mira y López, 1948).

Desde 1949 la orientación profesional formó parte de las políticas de gobierno en Argentina al ser incluida en la reforma constitucional como "complemento de la acción del instruir y educar" siendo "una función social que el Estado ampara y fomenta mediante instituciones que guíen a los jóvenes hacia las actividades para las que posean naturales aptitudes y capacidad, con el fin de que la adecuada elección profesional redunde en beneficio suyo y de la sociedad" (Nación Argentina, 1950, p. 23). Dos factores fundamentales habían contribuido al surgimiento de la orientación profesional: el conocimiento generado por la psicología, y especialmente la psicotecnia, respecto de las características personales necesarias para un buen desempeño laboral; y los cambios económicos y sociales efecto de la crisis de 1930, que exigieron nuevas herramientas de intervención, conformando el futuro campo de aplicación (Klappenbach, 2005). En 1985, el Ministerio de Educación y Justicia de la Nación, estableció la realización de orientación vocacional y ocupacional como incumbencia profesional de la psicología (Ministerio de Cultura y Educación, 1985). A lo largo del tiempo, la orientación profesional argentina fue enriqueciendo su corpus epistemológico e instrumental, desarrollando la línea de la orientación vocacional ocupacional, que consideraba tanto las habilidades e intereses del sujeto, como las posibilidades de inserción en el mercado laboral. El proceso de orientación es actualmente considerado como un proceso interpersonal entre orientador y orientado, en el cual interviene una amplia gama de problemáticas vinculadas a la elección de carrera, el ingreso, la adaptación y la aproximación a la ocupación que dicha carrera propicia dentro de una comunidad, con el inestimable aporte del campo de la educación (Stuart, 1999). Más allá de las diversas caracterizaciones y aportes de los cuales se fue nutriendo, la orientación profesional en Argentina siempre se ha caracterizado por su preocupación por los aspectos sociales, considerándolos parte ineludible y constitutiva de las elecciones educacionales y laborales que los individuos realizan. 


\section{Las condiciones socioeconómicas}

Si bien actualmente los indicadores socio-económicos demuestran una lenta recuperación, en Argentina existe aún una marcada inequidad social, con elevados índices de pobreza e indigencia, y altas tasas de desempleo. El último censo de 2002 indica que el desempleo fue del $17,8 \%$, hubo $57 \%$ de personas y $45,7 \%$ de hogares bajo la línea de la pobreza; $27,5 \%$ personas y $19 \%$ de hogares bajo la línea de la indigencia, y el ingreso per cápita fue de $\$ 434$ (aproximadamente $\$ 145$ dólares). Las cifras referidas a educación tampoco son alentadoras, ya que para esa fecha $4,1 \%$ de los adultos nunca había ido a la escuela, 35,1\% sólo había terminado la escolaridad primaria, 12,9\% había terminado estudios secundarios, y sólo el 7,7\% los estudios universitarios (Instituto Nacional de Estadísticas y Censos, 2002). A pesar de la lenta mejoría de algunos de estos aspectos, Argentina todavía padece severas condiciones estructurales de exclusión social, producto de las políticas de desregulación económica de la década del '90, y que seguramente llevará tiempo modificar (Ferrero, 2003a).

Frente a este tipo de situaciones, actualmente se plantea la necesidad de resignificar la aplicación de la orientación vocacional a la luz de las situaciones socioeconómicas imperantes, enfatizando la influencia que los factores de contexto tienen sobre los consultantes, y dado el rol fundamental que la ocupación juega en la identidad individual (Rísquez, 2004). Incluso numerosos estudios epidemiológicos demuestran la relación entre condiciones socioeconómicas y educacionales deficitarias y una mayor prevalencia de patología mental, debido a un contexto desventajoso (Ferrero, 2003b).

La situación política y económica producto de la globalización ha intensificado diversas contradicciones sociales que impactan directamente en la manera de concebir la educación, y especialmente en el ámbito de la orientación profesional. La crisis del Estado de bienestar, la desregulación del mercado laboral, y la dificultad para acceder a derechos básicos como salud y educación, han incrementado las desigualdades sociales, generando aún más tensión frente a una supuesta homogenización cultural en contextos crecientemente multiculturales, donde incluso resurgen los nacionalismos. Se declama una "sociedad de oportunidades", pero al mismo tiempo hay mayor pesimismo individual, con mayores niveles de conformismo social y de acomodación a condiciones de vida impuestas por el mercado. Por ello la orientación profesional debe trascender el dar información sobre puestos de trabajo y su adecuación a los mismos, e implicarse en la educación sociolaboral de una ciudadanía autónoma y responsable. La formación y orientación de ciudadanos y ciudadanas no implica su mera colocación laboral, sino que debe apuntar a una tarea de educación sociolaboral, que permita incorporarse a circuitos productivos, tanto como colaborar en la reconstrucción misma de esos circuitos y de la dimensión sociopolítica de la vida laboral. Para ello es fundamental que los orientadores no cedan a las presiones consumistas que los conminan a actuar acorde a exigencias del mercado (Fernández Sierra, 2006). Es fundamental que los procesos de orientación promuevan la inclusión de conceptos referidos al campo social, en tanto lugar hacia donde se dirige su aplicación incluso en procesos individuales de orientación. Para ello es indispensable considerar el futuro rol de la orientación frente a una sociedad signada por permanentes cambios, como los avances tecnológicos y la cambiante estructura etaria y multicultural de los pueblos (Benavent Oltra, 2003; Hiebert, 2005). El progreso científico tecnológico está en gran medida determinado por elementos sociales, valores morales, intereses profesionales, y condicionantes políticos y económicos, los cua- 
les a su vez se ven afectados por dicho proceso. En el ámbito de la formación específica, la tecnología debe ser incluida como objetivo y como contenido de la orientación profesional, generando un análisis crítico sobre las tecnologías en sí mismas y su utilización, y planteando los conflictos existentes entre los valores exigidos por la sociedad tecnológica y las telecomunicaciones y los valores propios de la formación curricular. Por ello, a la autonomía moral, la empatía y la reflexión, debe agregarse un cuarto elemento, el del compromiso social, especialmente en el caso de los educadores (Cortés Parcial, 2006). La formación en orientación profesional deberá entonces integrarse en los currículos en base a ciertos principios pedagógicos básicos: la corresponsabilidad interprofesional, el enfoque interdisciplinar, la perspectiva educativa y la proyección vital, apuntando no solamente a la resolución de problemas sino a la adquisición de una perspectiva moral, ya que las decisiones en el ámbito profesional y laboral implican muchas veces, un conflicto de valores (Cortés Parcial, 2006; Fernández Sierra, 2006). Por ello, frente a condiciones socioeconómicas adversas es indispensable poder advertir las motivaciones de consultantes y orientadores, y mantener los fines preventivos y de responsabilidad social de la orientación contextualizada (Gavilán, Quiles \& Chá, 2005; Royo, 2002).

\section{La responsabilidad social a partir de pautas éticas y competencias profesionales}

En Argentina, la preocupación por la responsabilidad social como parte inherente a los procesos de orientación, se advierte incluso en las múltiples referencias existentes en diversos códigos de ética de psicología a nivel nacional y regional, dado que es esta profesión la que tradicionalmente ha llevado a cabo la tarea de orientación en este país. Como muchos otros conceptos que hacen a una práctica profesional ética, la responsabilidad social se relaciona directamente con el respeto y la dignidad de personas y pueblos, ya que toda práctica profesional debe sustentarse, en primer lugar, en el ineludible respeto por los derechos humanos (Ferrero, 2006). En 1997, y a nivel regional, el Protocolo de Acuerdo Marco de Principios Éticos para el Ejercicio Profesional de los Psicólogos en el Mercosur y Países Asociados, vigente en seis países de Suramérica, incluida Argentina, establecía como principio básico del accionar científico y profesional de la psicología la Responsabilidad social, sosteniendo que: "Los psicólogos ejercen su compromiso social a través del estudio de la realidad y promueven y/o facilitan el desarrollo de leyes y políticas sociales que apunten, desde su especificidad profesional, a crear condiciones que contribuyan al bienestar y desarrollo del individuo y de la comunidad" (Comité Coordinador de Psicólogos del Mercosur y Países Asociados, 1997, p. 2). A nivel nacional, la Declaración de Principios del Código de Ética de la Federación de Psicólogos de la República Argentina, de 1999, incorpora lo establecido por dicho Protocolo de Acuerdo Marco, reiterándose el compromiso existente entre la profesión y la sociedad (FePRA, 1999). Más allá de la deontología de la psicología, las normas éticas de la Asociación Internacional de Orientación Educativa y Profesional, también señalan en diversos artículos la importancia de la responsabilidad social. Así, en el apartado referido a Responsabilidades Éticas con los Clientes, se expresa que los miembros de la Asociación deben respetar la dignidad y derechos de toda persona a la que se le presta un servicio educativo y profesional, y estar actualizados respecto de las leyes y políticas referidas a los derechos de los clientes $\left(\right.$ Art. $1^{\circ}$ ). En ese mismo apartado se señala que el orientador debe promover la igualdad de oportunidades en la orientación educativa y profe- 
sional sin ningún tipo de prejuicio hacia las personas debido a su condición social, evitando todo tipo de discriminación (Art $2^{\circ}$ ). La tarea debe ser sensible a las necesidades de los consultantes (educativas, vocacionales, personales y sociales) ya que interactúan y afectan la planificación o la adaptación en la educación y formación, ocupaciones y carreras (Art. $3^{\circ}$ ). Finalmente, se enuncia que los consultantes de grupos menos representados deben acceder igualmente a las mejores técnicas disponibles (Art. $7^{\circ}$ ). El apartado referido a Actitudes hacia al Gobierno y otras Agencias Comunitarias, establece que los orientadores propiciarán el desarrollo de servicios de orientación educativa y profesional que sean relevantes para los consultantes, en estrecha colaboración con políticos, legisladores o personal administrativo (Art $1^{\circ}$ ). Las Responsabilidades como Orientador Individual, destacan que la práctica debe reflejar tanto principios humanísticos que subyacen al comportamiento ético como la atención a aquellas cuestiones sociales y políticas cambiantes que tienen implicaciones para su práctica (Art. $3^{\circ}$ ). También este apartado incluye la necesidad de controlar y mantener las competencias profesionales y recibir formación continua para ser capaces de proporcionar sus servicios a consultantes de culturas diversas (Art. 4) (Asociación Internacional de Orientación Educativa y Profesional -AIOEP_, 1995).

Si consideramos las competencias específicas referidas al campo de la orientación, se advierte la importancia de la dimensión social en relación a las Competencias Internacionales para los Profesionales de Orientación y Educación, aprobadas en Berna por la Asamblea General de la Asociación Internacional de Orientación Educativa y Profesional (Asociación Internacional de Orientación Educativa y Profesional —AIOEP_, 2003). Estas competencias recomiendan utilizar herramientas de evaluación específicas suficientemente validadas, y mantener una formación continua, y una adecuada supervisión. Para responder a los desafíos actuales de la tarea de orientación, los profesionales deben demostrar capacidad en dos áreas: conocimientos y habilidades que fundamenten la praxis, y una toma de decisiones ética (Stuart, 1999). Las "Competencias Centrales" de los orientadores incluyen: demostrar profesionalidad y comportamiento ético en su desempeño; apreciar y atender las diferencias culturales de los consultantes, posibilitando la interacción efectiva con poblaciones diversas; poseer información actualizada sobre educación, formación, tendencias de empleo, mercado de trabajo y asuntos sociales; y contar con sensibilidad social e inter-cultural. Las "Competencias Especializadas" incluidas en este documento hacen referencia a competencias de diagnóstico, orientación educativa, desarrollo de la carrera, counseling, información, consulta, investigación, gestión de programas y servicios, desarrollo humano y empleo. En la mayor parte de estos grupos de competencias se destacan numerosas referencias a la responsabilidad social inherente a la tarea de orientación. Así, en las "Competencias de Diagnóstico", se indica especialmente llevar a cabo un análisis del contexto en el que está inmerso el cliente; y en la "Competencias de Desarrollo de la Carrera", se señala la importancia de identificar los factores implicados (familia, amigos, oportunidades educativas y financieras) y actitudes sesgadas (sobre género, raza, edad y cultura) en el proceso de toma de decisiones, tanto como de conocer los servicios de asistencia e información de empleo y economía, y poseer habilidad para utilizar los recursos de la carrera diseñados para cubrir necesidades especiales de grupos específicos (migrantes, grupos étnicos y población en riesgo). En las "Competencias de Información", se incluye la conveniencia de recopilar, organizar y distribuir información actualizada sobre el desarrollo personal, social y de la carrera. Las "Competencias de Gestión de Programas y Servicios" indican identificar las poblaciones objeto del estudio, promoviendo en ellas el interés comunitario sobre el progra- 
ma o servicio en curso. Las "Competencias de Desarrollo Comunitario" destacan la importancia de establecer contacto con miembros de la comunidad y analizar sus necesidades, recursos humanos y materiales, haciendo uso efectivo de éstos acorde a dichas necesidades, desarrollando planes de acción con miras de mejora económica, social, educativa y del empleo, y en un marco de cooperación con instituciones nacionales e internacionales de orientación educativa y profesional (Asociación Internacional de Orientación Educativa y Profesional - AIOEP-, 2003).

\section{Conclusiones}

La situación política y económica imperante en numerosos países, incluida la Argentina, ha generado un paulatino incremento de tensiones en el entramado social: desempleo, falta de oportunidades, desigualdades sociales, y dificultad para acceder a derechos básicos como la salud y la educación. Ello ha aumentado sensiblemente la exclusión en un doble sentido: hay cada vez mayor cantidad de actores sociales excluidos y con un mayor nivel de exclusión. Esta circunstancia ha repercutido directamente en el ámbito educativo y laboral, y, por tanto, en el campo específico de la orientación profesional. Frente a este panorama se impone en estos países que la orientación profesional renueve cotidianamente su compromiso, incluso a partir de lo establecido por diversos códigos de ética profesionales nacionales y regionales, y por las indicaciones de las mismas competencias disciplinares de la orientación profesional a nivel internacional, ambos aspectos vigentes en Argentina. El mencionado compromiso se sustenta en la convicción de que la orientación de la ciudadanía es básicamente una tarea de educación sociolaboral, que ya va mucho más allá de la inclusión del individuo a un determinado circuito productivo, en tanto implica también a la reconstrucción misma de la dimensión sociopolítica de la vida laboral. Promover la consideración de aspectos socio-económicos en la tarea de orientación permite evitar contribuir involuntariamente con los circuitos de exclusión social, colabora en el procesamiento del impacto del progreso científico-tecnológico de modo tal que no genere aún mayor desigualdad, y propicia la reconstrucción de aquellos entramados sociales dañados por las distintas formas que adquiere la inequidad. Esta tarea de reconstrucción permitirá contribuir al desarrollo de individuos más autónomos y capaces de advertir tensiones, y posibles soluciones, entre sus propios valores y los de una sociedad que no siempre los considera como parte de sí.

\section{Referencias bibliográficas}

Asociación Internacional de Orientación Educativa y Profesional. —AIOEP_ (1995). Normas Éticas de la Asociación Internacinal de Orientación Educativa y Profesional. Estocolmo. Autor.

Asociación Internacinal de Orientación Educativa y Profesional - AIOEP- (2003). Competencias Internacionales para los Profesionales de Orientación y de Educación. Berna. Autor.

Benavent Oltra, J. (2003). "Reflexiones sobre el futuro de la orientación psicopedagógica inmersa en una encrucijada sociocultural”. Revista española de orientación y psicopedagogía, 14 (1), 41-59.

Comité Coordinador de Psicólogos del Mercosur y Países Asociados (1997). Protocolo de acuerdo marco de principios éticos para el ejercicio profesional de los psicólogos en el Mercosur y Países Asociados. A psicologia no Mercosul (pp. 11-14). Brasilia. Autor. 
Cortés Pascual, P. (2006). "Un análisis de la orientación profesional desde la tecnoética educativa". Revista española de orientación y psicopedagogía, 17 (2), 181-193.

Federación de Psicólogos de la República Argentina (1999). Código de Etica. Buenos Aires. Autor.

Fernández Sierra, J. (2006). "Educación para la carrera y globalización ¿atrapados en las contradiccciones sociolaborales neoliberales?" Revista española de orientación y psicopedagogía, 17 (2), 261-272.

Ferrero, A. (2003a). "Consecuencias sociales y psicológicas de la globalización en Argentina. Una mirada desde la ética profesional". Revista Iberoamericana de Educación, Salud y Trabajo, 4, 253-264.

Ferrero, A. (2003b). "Prevalencia de patología mental grave en la Ciudad de San Luis". Vertex. Revista Argentina de Psiquiatría, 14, 11-18.

Ferrero, A. (2006). "Human Rights and Psychology Ethics Codes in Argentina". In A. Columbus (Ed.), Advances in Psychology Research, 41, pp. 129-135. New York. Nova Publishers.

Gavilán, M., Quiles, C. \& Chá, T. (2005). "Paradoja: alta problemática psicosocial - baja intervención orientadora". Orientación y Sociedad, 5, 141-156.

Hiebert, B. (2005). "Perspectivas en Orientación e inclusión de lo social en una sociedad globalizada". Orientación y Sociedad, 5, 11-22.

Instituto Nacional de Estadísticas y Censos (2002). Encuesta permanente hogares. Buenos Aires. Autor.

Klappenbach, H. (2005a). "Historia de la orientación profesional en Argentina". Orientación y Sociedad, 5, 37-48.

Ministerio de Cultura y Educación (1985). Resolución 2447/85 [Incumbencias de la Psicología]. Autor.

Mira y López, E. (1948). Manual de Orientación Profesional. Buenos Aires. Kapelusz.

Nación Argentina (1950). “Constitución Nacional de 1949”. Digesto Constitucional de la Nación Argentina (pp. 7-24). Buenos Aires. Congreso de la Nación.

Rísquez, A. (2004). "Efectos de las diferencias interculturales sobre el desarrollo de la carrera y la orientación vocacional: un estudio empírico de los factores que influyen en la motivación hacia el trabajo de trabajadors irlandeses y españoles". Revista española de orientación y psicopedagogía, 15 (1), 11-31.

Royo, F. (2002). "Resumen de desempeño profesional de los orientadores de los institutos de educación Secundaria de Salamanca. Creación de un instrumento de valoración y autovaloración". Revista española de orientación y psicopedagogía, 13 (1), 31-50.

Stuart, J. (1999). "Ethical Issues in Career Counseling”. Guidance \& Counseling, 14 (2), 18-22.

Fecha de recepción: 09-03-2007

Fecha de revisión: 03-12-2008

Fecha de aceptación: 18-02-2009 